\title{
Guidance for Evaluating the Safety of Experimental Releases of Mosquitoes, Emphasizing Mark-Release-Recapture Techniques
}

\author{
Mark Q. Benedict, J. Derek Charlwood, Laura C. Harrington,' L. Philip Lounibos, \\ William K. Reisen, and Walter J. Tabachnick ${ }^{6}$
}

\begin{abstract}
Experimental releases of mosquitoes are performed to understand characteristics of populations related to the biology, ability to transmit pathogens, and ultimately their control. In this article, we discuss considerations related to the safety of experimental releases of living mosquitoes, applying principles of good practice in vector biology that protect human health and comfort. We describe specific factors of experimental releases of mosquitoes that we believe are critical to inform institutional biosafety committees and similar review boards to which proposals to conduct mosquito release experiments have been submitted. In this study, "experimental releases" means those that do not significantly increase vector capacity or nuisance biting relative to the unperturbed natural baseline. This document specifically does not address releases of mosquitoes for ongoing control programs or trials of new control methods for which broader assessments of risk are required. It also does not address releases of transgenic or exotic (non-native) mosquito species, both of which require particular regulatory approval. Experimental releases may include females and males and evaluation must consider their effects based on the number released, their genotype and phenotype, the environment into which they are released, and postrelease collection activities. We consider whether increases of disease transmission and nuisance biting might result from proposed experimental releases against the backdrop of natural population size variation. We recommend that experimental releases be conducted in a manner that can be reasonably argued to have insignificant negative effects. Reviewers of proposals for experimental releases should expect applicants to provide such an argument based on evidence from similar studies and their planned activities. This document provides guidance for creating and evaluating such proposals.
\end{abstract}

Keywords: biosafety, disease vector, ecology, population biology

\section{Introduction}

I N ADDITION TO NUISANCE BITING, mosquitoes are important vectors of agents of human diseases, including filariasis, dengue, yellow fever, chikungunya, Zika, encephalitis, and malaria (www.mosquito.org/mosquito-borne-diseases). They also transmit pathogens to animals, including filarial worms (e.g., Dirofilaria immitis), Rift Valley Fever, and West Nile virus, which can cause illness in both animals and humans.
Despite the importance of mosquitoes, many of their bionomic and behavioral characteristics remain poorly known. This is due, in part, to their small size, difficulty in trapping them efficiently, variable abundance temporally and spatially, the nocturnal habits of many species, and the difficult to reach environments in which many occur.

Numerous methods for conducting field studies of mosquitoes have been devised (Silver 2007), and despite much effort, even the best are often crude and inaccurate, particularly

\footnotetext{
${ }^{1}$ Entomology Branch, Centers for Disease Control and Prevention (CDC), Atlanta, Georgia.

${ }^{2}$ Centre for Health Research and Development, Faculty of Life, University of Copenhagen, Copenhagen, Denmark.

${ }^{3}$ Department of Entomology, Cornell University, Ithaca, New York.

${ }^{4}$ Florida Medical Entomology Laboratory, University of Florida, Gainesville, Florida.

${ }^{5}$ Center for Vectorborne Diseases, Department of Pathology, Microbiology, and Immunology, School of Veterinary Medicine, University of California, Davis, Davis, California.

${ }^{6}$ Florida Medical Entomology Laboratory, University of Florida, IFAS, Vero Beach, Florida.
}

(c) Mark Q. Benedict et al. 2018; Published by Mary Ann Liebert, Inc. This is an Open Access article distributed under the terms of the Creative Commons Attribution License, which permits unrestricted use, distribution, and reproduction in any medium, provided the original work is properly cited. 
for estimating population size. This document introduces the reader to one method for doing this, Mark-Release-Recapture (MRR). This summary particularly emphasizes characteristics of releases that might influence decision-making and design of MRR studies that would affect biosafety and provide guidance for evaluating their safety and practices to reduce possible risks. It is not a comprehensive review, and articles cited herein have been selected from an extensive literature to demonstrate examples of research tools and results. Space does not permit coverage of analytical methods on MRR data, which have been the topics of entire books (Amstrup et al. 2010). This overview applies to MRR of adult mosquitoes, although the considerations are applicable to aquatic immature stages.

This discussion complements the efforts of Achee et al. (2015) who described risks related to another method used in vector biology, "human landing catches." Although possibly relevant for MRR investigations, we will not revisit the issues of informed consent and worker safety discussed there.

Issues related to releases of genetically modified mosquitoes are likely to be considered partly by institutional biosafety committees but are expected to involve additional review by regulatory authorities; thus researchers are encouraged to consult applicable legislation in this regard. Guidance for releases of genetically modified mosquitoes has been developed in another document under the leadership of the Foundation for the National Institutes of Health and the World Health Organization (WHO and FNIH 2014).

We do not address methods for producing and containing mosquitoes being produced for release. These are addressed by national regulation, generally following principles similar to those articulated in the Arthropod Containment Guidelines (ACME-ASTMH 2003). Depending on whether a vector is present at the site of production, either level 1 or level 2 containment is recommended.

There are two hazards of most importance that might result from experimental releases of mosquitoes: a transient or stable increase in the mosquito population that results in increased nuisance biting or disease transmission; and a change in the phenotype of the wild population that makes it more difficult to control or increases its ability to transmit disease (vector capacity). Both of these are outcomes against which appropriate measures should be taken.

\section{What Is MRR?}

Regardless of the mosquito species and pathogen(s) of interest, the characteristics of mosquitoes that determine their likelihood to transmit disease are known as their vector capacity. Vectorial capacity for a particular human disease agent is determined by longevity (daily survival), likelihood of becoming infected with a particular pathogen, propensity to feed on either humans or a particular animal as appropriate for the agent, incubation period of the agent, and female abundance. Malaria models are reviewed in Mandal et al. (2011). Dispersal and spatial heterogeneity have also been identified as additional factors to consider in disease transmission (Perkins et al. 2013).

Due to the constraints of size, temperature, humidity, and habitat simplicity of laboratory studies, wild population abundance, dispersal, and daily survival are impossible to estimate without field studies. These characteristics can vary dramatically, geographically, and temporally. Models and laboratory studies are useful tools, but field studies of these factors are essential to parameterize and validate models to plan effective control of mosquitoes.

All three contributors to vectorial capacity can be estimated in a single experiment method using MRR. This consists of releasing marked mosquitoes into a wild population and, usually over a period of several days, recovering as many adults of the target species as is practical from which the frequency of marked mosquitoes is determined. This technique has been used for species as diverse as birds, whales, and insects (Amstrup et al. 2010). In essence, the size of the wild population is estimated by the degree of dilution of a known number of marked mosquitoes into the unmarked wild population, whose numbers are unknown, and subsequent measurement of the dilution factor by captures.

Occasionally, the releases and recaptures are performed in a spatial pattern that allows one to determine movement of marked individuals. The number of recaptures over time and space and the proportion of marked mosquitoes among those captured can provide estimates of longevity, migration, and abundance of the wild population. The calculation of these characteristics depends upon several assumptions, but MRR has proven to be one of the most useful tools of vector biology.

Three outcomes are critical to measure in typical MRR experiments: how many of the marked and unmarked wildtype mosquitoes are captured, when and where. While the percentage of marked adults that are recaptured does not necessarily influence the interpretation of the results, it can indicate the thoroughness of the recapture efforts and the sensitivity of the capture methodology; therefore it is of secondary interest as an outcome which is usually reported.

Guerra et al. (2014) collected published data, analyzed it, and systemized the information on mosquito MRRs since the method was first applied to mosquitoes. They summarized this information and have also made their database available upon request. The releases they reported consisted of females, the sex that is capable of transmitting disease agents. Because males are not directly involved in disease transmission, they are seldom released, and when they are, it is often in the context of genetic control techniques such as the sterile insect technique in which only males are typically released (Dame et al. 2009).

The number of mosquitoes required for releases is usually large (Fig. 1) owing to a low probability of recapture. There are no highly sensitive methods to collect most species of adult mosquitoes, and average values of recaptures range from a high of $8 \%$ for Aedes spp., $4 \%$ for Anopheles spp., and $1 \%$ for Culex spp. (Guerra et al. 2014).

While the thousands of mosquitoes typically released in MRR may seem large, a sense of proportion is provided by the numbers of wild-type mosquitoes that are typically captured along with the marked ones during the postrelease collection activities. This number is usually much larger than the number released meaning that most MRR activities result in a net reduction in the mosquito population. In the extensive database of Guerra et al. (2014), in the releases for which recapture size was reported, the size of an average release of females was 8,824 . The average number of females recaptured was 25,556 (Fig. 1). In 81 of 120 (67.5\%) of these releases the number of captured females was greater than the number released. In a handful, no report of captures was 


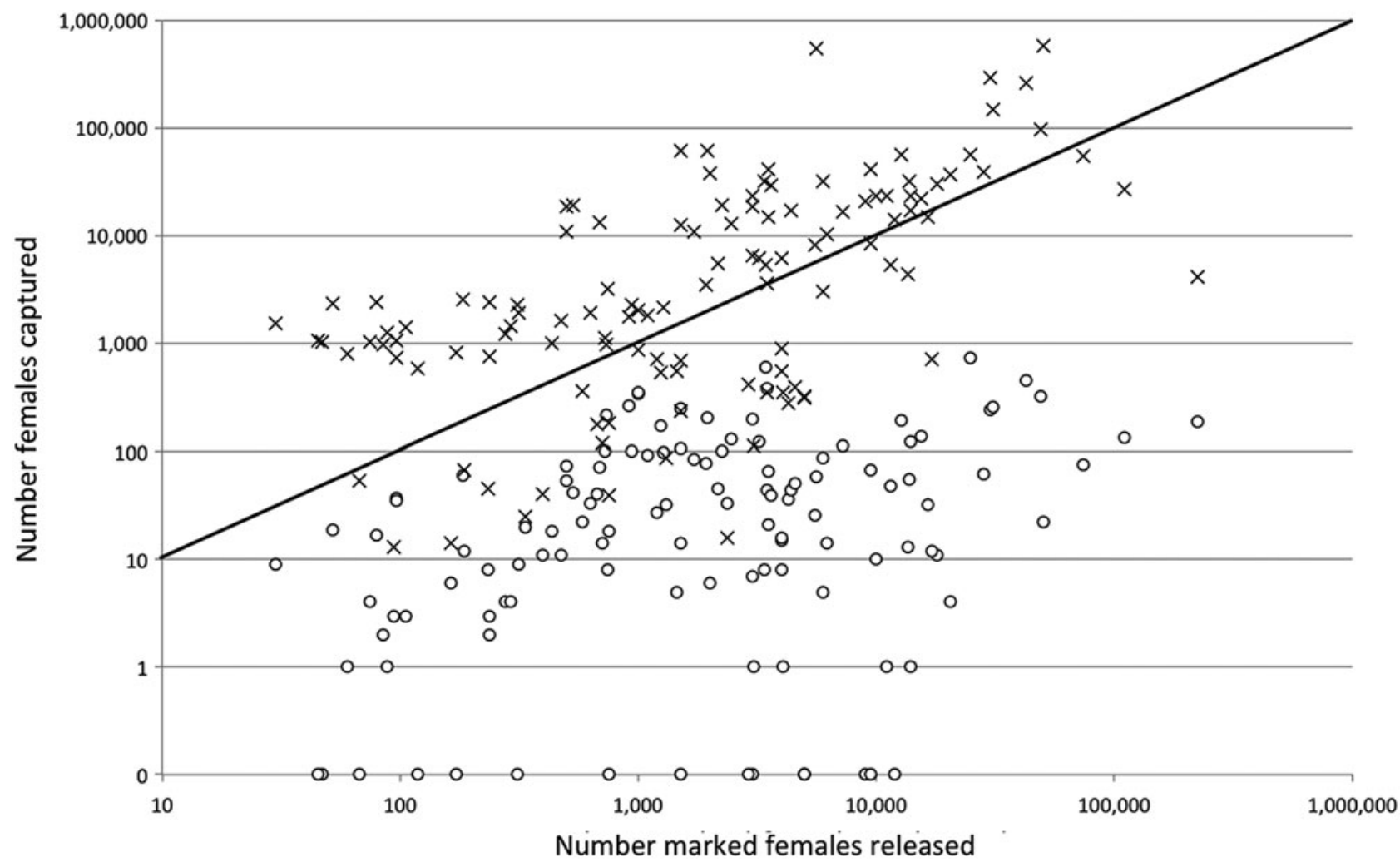

FIG. 1. Relationship of releases to captures. Numbers of marked $(\bigcirc)$ and wild-type $(\times)$ females recovered in the compilation of mosquito releases of Guerra (2014). Symbols for wild-type females above the diagonal line represent MRR activities in which the number of unmarked females captured was greater than the number of marked females that were released resulting in an overall reduction in the population size as a result of the MRR. MRR, Mark-Release-Recapture.

made, so it is possible that the proportion of efforts accomplishing population reductions could have been greater with even a modest effort. Overall, the contribution of released mosquitoes to the wild population is negated by the intensive postrelease efforts to capture adults, and this is an important safety consideration.

\section{Considerations and Assumptions Underlying MRR}

There are a number of assumptions that apply to interpretation of MRR experiments. Briefly:

(1) Marking techniques should not adversely affect the insects so that they will behave normally following marking; for example, "dusted" individuals may have an initial "shock" reaction and are sometimes held for a day before release.

(2) For the purpose of estimating population size, the marked insects are assumed to be distributed randomly in the population before recapture and so all have the same chance of being caught (although there are a number of models that accommodate this confounding factor).

(3) The mark is not lost (or overlooked) during the experiment.

(4) The initial sample and the subsequent samples are independent of one another.

An important distinction exists between "open" and "closed" populations with respect to MRR studies. A closed population is one in which there is neither immigration or emigration nor birth or death during the study; the population remains constant in size and composition during the study. An open population is one in which insects enter and leave through any of the above processes. Migration is occasionally minimized as a significant confounding variable by choosing wild-type populations that are expected to be isolated by some geographic (e.g., water or mountain) or ecological features (e.g., desert, forest, fields, or human dominated settings for peridomestic species). Such isolating mechanisms can contribute to a population behaving as closed.

\section{The source of mosquitoes for release}

Adults to be released can be obtained as field-caught individuals, progeny of field-caught adults, or laboratoryreared colonized mosquitoes. The first two sources have the advantage that insects that are genetically similar to the unmarked population are released, minimizing concerns about laboratory selection for behaviors different from the population into which they are being released. Disadvantages include the fact that the age of insects upon release is sometimes unknown and numbers are generally smaller, while colonized mosquitoes have the advantage that mosquitoes of uniform age can be released in larger numbers. Due to their greater longevity and ease of capture, field-caught adults often consist mostly of females. Passive capture techniques, such as aspiration of resting adults from surfaces, can yield a more representative sex ratio. 
When abundant larval populations can be identified, these can be collected and reared to adulthood in the laboratory. Alternatively, F1 progeny of wild-caught females can be raised to adulthood. These sources provide genetically representative samples from the population of interest, and the differences between the released and wild mosquitoes are limited to the effects of larval culture and marking. There are issues to be considered however. According to the question being asked, different emergence times resulting from the range of larval stages collected preclude the use of some marking techniques due to a limited range of marking options (e.g., dusts only come in a few colors although this can be extended by blending). It has also been demonstrated that laboratory culture, even in the absence of selection, for unidentified reasons may have an effect on assortative mating (Aboagye-Antwi and Tripet 2010) or due to different sizes resulting from relatively high larval nutrition.

Adults from laboratory colonies are often used. Since it is relatively easy to produce adequate numbers that are of a similar age for releases, this is usually simpler and more predictable than using field-collected mosquitoes. However, the lower genetic relatedness to the wild population and laboratory selected behavioral characteristics of colonized mosquitoes possibly introduce biases due to genetic bottlenecks and selection for traits compatible with laboratory culture that may differ from the populations of interest (e.g., reduced flight, mating behavior, and change in survival rate). These differences can confound accurate interpretation of the MRR results.

\section{Marking}

In his valuable compilation of mosquito ecology methods, Service (revised and edited by Silver 2007) divided marking methods into five classes: stains (including paints); dusts and powders; trace elements; radioactive materials; and phenotypic mutants. By far, the most commonly used marking is fluorescent "DayGlo" type dust (see Figure 6 of Guerra et al. 2014 and Fig. 2, this article). This is applied to adults usually by suspending it in the air of the adult container where it clings to the adults. It is particularly useful because large numbers of adults can be marked and examined en masse, whereas some methods require marking and examination of individuals-sometimes in thousands. Some studies have involved applying a small drop of paint to the thorax of mosquitoes (Harrington et al. 2005, 2008, Lehmann et al. 2010). This approach can be useful if multiple marks are required and can be combined with fluorescent powder dusting. An experienced person can apply paint to hundreds of mosquitoes in a day.

More novel methods for marking adults have been proposed or tested. Marking methods widely used on other insects, such as spraying with a proteinaceous solution detectable by enzyme-linked immunosorbent assay (e.g., Hagler et al. 2009), have apparently not yet been applied to mosquitoes although there is no obvious reason that it would not be possible. For dispersal studies of larger insects such as dragonflies and bees, radio transmitters have revolutionized tracking (e.g., Wikelski et al. 2014). Eventually, progress in miniaturization of electronics may allow for radio tracking of mosquitoes. The introduction of a unique host with a readily identifiable blood into a village in Papua New Guinea enabled Charlwood et al. (1985) to estimate the distance flown by determining the blood source of engorged mosquitoes after blood feeding.

For some techniques, marking in one life history stage persists into another, in which the mark may be detected. For example, Honório et al. (2003) fed Aedes aegypti females bloodmeals spiked with the alkali metal rubidium (as $\mathrm{RbCl}$ ), which was subsequently detected in eggs laid by gravid females in the field in Rio de Janeiro, Brazil. Attempts to mark Culex quinquefasciatus larvae with various dyes combined with the larval food and one combination of blue dyes were detectable in $97 \%$ of the adults; however, the number in which the mark could be detected diminished with age (Welch et al. 2006).

\section{Recaptures}

After releases, mosquitoes may be captured with animal or human baits, traps, or by aspiration of individuals from resting sites (e.g., Frank and Curtis 1977, Guerra et al. 2014). If live captures are required, it is imperative that the collection method should not harm them. Numerous novel methods

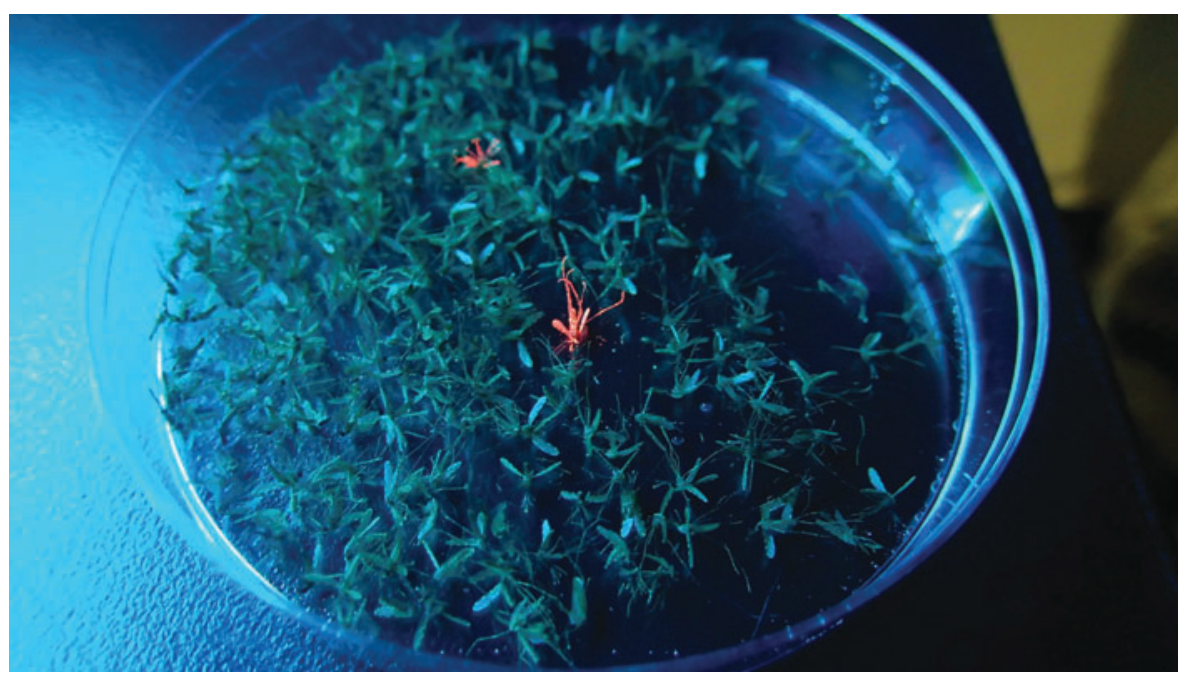

FIG. 2. Photograph of dusted mosquitoes. Two Anopheles gambiae adults that were marked with red fluorescent powder (No. 1162; Bioquip, Dominquez, CA) are visible in this dish. In this case, the illumination consists of a benchtop ultraviolet tube source (UVP, Upland, CA); however various battery powered UV and near-UV LED sources are widely available, inexpensive, and are suitable for field use. UV, ultraviolet. 
for capture of all stages of mosquitoes have been compiled (Silver 2007). This extensive volume (1600 pages) is testament to the creativity of those devising methods for mosquito collection-and the difficulty of doing so.

\section{Dispersal techniques and distances}

All estimates of mosquito dispersal by MRR measure distances traveled between release and recapture points. As distance from the release site increases and the density of released mosquitoes declines, the number of sampling locations must increase. Since dispersal in insects is often leptokurtic (i.e., many insects dispersing less distance than expected from a normal distribution and a few dispersing further than expected), it is important to distinguish between mean and median flight range and maximum flight range.

\section{Survivorship}

If recapture efforts continue for a number of days after releases of mosquitoes of known age, estimates of survivorship can be calculated directly from recapture rates or indirectly, using models. As the same data set is used, it is commonplace for dispersal and survivorship to be estimated from the same study (e.g., Trpis and Hausermann 1986, Charlwood et al. 1988). Dispersal out of the study area cannot be distinguished from mortality and so it is useful to have independent estimates to support either estimate as was done by Gillies and Wilkes (1965), Charlwood (1986), and more recently by $\mathrm{Gu}$ et al. (2011).

\section{Population size}

Population size may be estimated with MRR data similar or identical to those used to calculate dispersal and survivorship; hence, several of the previous citations on MRRs with Ae. aegypti also estimate population size (e.g., Sheppard et al. 1969, McDonald 1977a, Trpis and Hausermann 1986). Population sizes estimated with the Jolly method on Ae. aegypti in coastal Kenyan villages (Lorimer et al. 1976) fell within the range of Lincoln Index (a more simplified calculation method) estimates applied to MRR data from a different year (McDonald 1977b).

\section{Examples of MRR studies}

- In a landmark study, Gillies (1961) individually marked 132,000 progeny of field collected Anopheles gambiae females and laboratory colony adults with poster paints and ${ }^{32} \mathrm{P}$ and $/$ or ${ }^{35} \mathrm{~S}$ radioisotope marking of larvae. He described survivorship and dispersal in both male and female mosquitoes as functions of age, wind, and human density.

- Domestic Ae. aegypti was tracked within and between villages on the Kenya coast, where prerelease females were marked with fluorescent dusts (McDonald 1977a) or marked individually with paints (Trpis and Hausermann 1986). Inter-village dispersal fell sharply beyond 200 meters, and most intra-village dispersal occurred within 20 meters of release sites (McDonald 1977a). Within villages, individual mosquitoes visited a maximum of five houses, but usually only one or two during the study period (Trpis and Hausermann 1986).
- In suburban Rio de Janeiro, gravid females Ae. aegypti and Aedes albopictus flew as much as 800 meters in 6 days to deposit Rb-laden eggs (Honório et al. 2003).

- Charlwood et al. (1988) tested the hypothesis that Anopheles farauti could recognize their region of origin in Papua New Guinea and showed that marked females were more likely to disperse if released in a non-native area compared to their natal village.

- MRR was used successfully to ascertain the source locations of pest mosquitoes in residential areas of Polk County, Florida by measuring distances and directions traveled of marked female mosquitoes originally captured in mechanical light traps (Morris et al. 1991).

- Using paint markings to identify individual Ae. aegypti, which were subsequently re-released after capture in houses, McDonald (1977b) estimated a daily survivorship of 0.89 for females and 0.77 for males in coastal Kenyan villages. These estimates are slightly higher than those of Sheppard et al. (1969) who applied the Fisher-Ford model to MRR data on the same species in a Buddhist temple in Bangkok, Thailand.

- Survivorship of large versus small individuals of $A e$. aegypti was compared among cohorts marked, released, and recaptured in Rio de Janeiro, Brazil, in which smaller males, but not females, had lower survivorship rates (Maciel-De-Freitas et al. 2007).

- Incorporating meteorological conditions into a nonlinear, least-squares model applied to MRRs of Culex pipiens in the Washington, DC area, Jones et al. (2012) showed that rainfall negatively impacted daily survivorship estimates and did not significantly differ between 2 successive years of MRR experiments.

- MRR applied to mosquitoes of the Anopheles punctulatus Group in Papua New Guinea detected no differences in survivorship estimates for three sibling species (Charlwood et al. 1986).

- MRR was used to demonstrate that availability of oviposition sites influenced gravid female Ae. aegypti movement in Puerto Rico (Edman et al. 1998).

As population size estimates would be compromised by significant migration, estimates with MRR have most often been applied to mosquito species with limited dispersal, such as container-inhabiting species. Trpis (1973) used MRR to estimate a total adult population size of 3,459 for Toxorhynchites brevipalpis, whose immatures occupied containers in a onehectare automobile dump near Dar Es Salaam, Tanzania.

One of the difficulties with MRR experiments meant to estimate population size can be that population size estimates are extremely sensitive to the effects of low recapture rates. For example, in the simplest case, suppose 100 insects are marked and released and of 100 recaptured one marked insect is recaptured. This would indicate a wild population size of 10,000 but if two marked insects had been recaptured the estimate would now be 5,000 and if three marked insects had been recaptured it would drop to 3,333 !

\section{Physiological states}

By examining recaptures for specific physical or internal attributes, which are associated with physiological states, MRR may be used to infer the timing of certain life history 
transitions in nature. Lounibos et al. (1998) released marked, unmated Anopheles darlingi females in a village near Porto Velho, Brazil and dissected recaptures to determine insemination status. Older females were more likely to have been mated, but even a few females $<2$ days old had been inseminated, probably without swarming.

Gillies and Wilkes (1965) carried out MRR on An. gambiae in Tanzanian villages to correlate calendar and physiological ages. Based on ovarian dissections of recaptured females, they concluded that the first gonotrophic (oviposition) cycle lasted 3-4 days but subsequent cycles only 3 days.

Estimates of the duration of the oviposition cycle of the different members of the An. farauti complex in Papua New Guinea (Charlwood et al. 1986) and An. darlingi in Brazil (Charlwood and Alecrim 1989) were obtained by bloodfeeding host seeking insects, marking, releasing, and recapturing them when they returned to feed. Species with more permanent, nearby larval habitats had a shorter cycle than those with temporary or distant sites. The duration of the cycle was also affected by moon phase, being shorter on nights just before the full moon than at other times (Birley and Charlwood 1989).

Based on the gut contents and age of recaptured Ae. ae gypti near Dar es Salaam, Tanzania, McClelland and Conway (1971) deduced that many females consumed blood twice during a single gonotrophic cycle.

\section{Behavioral studies}

MRR has also been used to determine if populations of Anopheles koliensis and An. punctulatus collected biting indoors or outdoors in one gonotrophic cycle continued to do so in subsequent iterations (Charlwood et al. 1986). They also determined that the duration of the oviposition cycle in mosquitoes fed late in the night was the same as that in mosquitoes that fed early in the night. Similarly Charlwood and Alecrim (1989) observed similar recapture times and rates among An. darlingi released engorged or unfed indicating that obtaining a bloodmeal did not affect survival in their study population.

Although, in general, a high recapture rate is necessary for an adequate interpretation of MRR data, in exceptional circumstances even a single recapture can provide information that can only be inferred by other methods. An example would be the recapture of a single An. gambiae 7 months after release in Mali that demonstrated survival (aestivation) of this species through the long dry season (Lehmann et al. 2010).

It should also be noted that MRR experiments are specific to the environment where they take place so that, for example, the same species may have different dispersal rates according to the nature of the terrain and distribution of hosts, larval habitats/oviposition sites. This limits definitive extrapolation of the conclusions of a single MRR study across all habitats.

\section{Effects of Experimental Releases on Mosquito Population Sizes}

Because of the anticipated low recapture rates and sensitivity of population size estimates to the numbers recaptured, large numbers of marked females (and males) are usually released during MRR experiments. The numbers released typically represent only a small fraction of the actual size of the target population as estimated using procedures such as Bailey's (1952) modification of the Lincoln Index. As summarized above, most MRR experiments release far fewer adult mosquitoes than are removed during subsequent sampling to recapture the marked adults. For example, 38,736 female and 45,504 male laboratoryreared Culex tritaeniorhynchus were released at a village along the Ravi River near Lahore, Pakistan (Reisen et al. 1978); however, 113,838 and 38,981 unmarked females and males, respectively, were removed during recapture attempts. These were only a small portion of the estimated wild population of 3,520,000 females and 3,570,000 males in the study area. Similarly, 19,419 laboratory-reared female Culex tarsalis were released in Coachella Valley, California to estimate dispersal, survivorship, and population size, and 115,050 females were removed by sampling from an estimated wild population of 914,000 females (Reisen and Lothrop 1995). Several experiments in Florida released ca. 250,000 marked Culex nigripalpus females, with 3,117,237 unmarked females being removed during recapture attempts (Nayar and Provost 1980). In contrast, following the release of 583,000 adult $C x$. quinquefasciatus emerging from larval containers treated with ${ }^{32} \mathrm{P}$ in Rangoon, Burma, 46,275 unmarked adults were removed during recapture attempts from an adult population estimated to range between 42 and 87 million (Lindquist et al. 1967). This did not result in a net population increase because the mosquitoes that were marked originated from natural populations and it illustrates the exception that in some instances recaptures do not exceed the number marked.

In the above experiments, daily estimates of population size were conducted immediately following release and were performed too soon to detect lagging changes that might have occurred in the next generation; however, seasonality and weather strongly dictate population size. For example, the second release conducted in the Coachella Valley estimated a two order of magnitude decrease in population size (Reisen and Lothrop 1995), far greater than what could have been caused by the number of adults released. Similarly, a series of intensive monthly release-recapture experiments in cotton fields along the Kern River in California (Reisen et al. 1992) showed that changes in female abundance were related to agricultural practices and not due to the release of adult $C x$. tarsalis.

Typically, smaller numbers have been used for studying the population ecology of indoor resting Anopheles and Aedes species. For example, in Punjab Province, Pakistan, following the release of 1,623 marked Anopheles culicifacies females, 3,331 unmarked females were removed during recapture attempts from an estimated population size earlier estimated at 2,609 females (Reisen et al. 1981). This paradoxical result was explained by high immigration rates into the primary resting site used for sampling. Similar results were found with Ae. aegypti (Trpis et al. 1995), with 338 females released and 538 unmarked females captured from an estimated daily population of 365 females.

In conclusion, as opposed to large-scale mass releases attempting population control, release of adults for MRR studies consists of such small numbers that they have little impact on the size of the endemic population within the study area due to much larger size and rapid replacement. 
Relative reproductive isolation of released demes (populations of potentially interbreeding individuals of the same species) may result in the failure of their specific genotypes to become readily incorporated into the target population of a different genotype (Reisen et al. 1985). Possible exceptions might occur for small closed and isolated populations that are not limited by ecological conditions and where the released adults are the same genotype as the target population. Here the pulse of released adults might exploit available habitats and create a temporary population increase until natural ecological factors once again limit abundance.

\section{Alternatives to Releases of Mosquitoes}

Some characteristics of natural mosquito populations that are often determined by MRR can be estimated by other methods, particularly the relative abundance of wild populations and migration. An estimate of the population's genetic complexity has a relationship to population size. Population genetic methods allow estimations of the "effective population size" $\left(\mathrm{N}_{\mathrm{e}}\right)$. This is an estimate of the population size making certain assumptions about genetic drift, random mating, and inbreeding. It has been applied to mosquito populations, and in one series of studies, the estimates of population size were compared with estimates based on MRR (Taylor and Manoukis 2003). Because $\mathrm{N}_{\mathrm{e}}$ is based on a hypothetical population and does not always reflect what is often rapid and dramatic population fluctuations, comparison with estimates of population size made by other methods is still needed. For example, Taylor and Manoukis (2003) estimated $\mathrm{N}_{\mathrm{e}} \sim 10$-fold below that of the peak population size measured by MRR. While $\mathrm{N}_{\mathrm{e}}$ can provide a general view of the wild population size, it is not suitable for real-time estimates of absolute size fluctuations.

The effective migration rate can also be estimated from population genetic analysis. For genetic control efforts, this knowledge is valuable since it describes the potential for movement of genetic material between populations such as might occur during attempts to control mosquitoes.

Relative abundance of mosquitoes can be determined by any of several collection methods such as trapping, aspiration, and insecticide knockdown. But even such systematic regular surveys of larvae or adults provide only relative indices of temporal changes in seasonal abundance; and as noted, current collection methods are neither efficient nor necessarily representative of the natural population. In the absence of an external reference such as MRR that "calibrate" the relative abundance indices, it is impossible to extrapolate such measures to absolute population sizes.

\section{Guidance for Evaluating the Safety of MRR Experiments}

\section{Potential effects of typical releases on disease transmission}

Male releases. Males have occasionally been released in the context of genetic control projects and were not considered by Guerra et al. (2014). Considering that ratios of males to females at eclosion are approximately equal, the abundance of male mosquitoes is assumed not to limit mosquito population reproductive capacity since males can mate sev- eral females, and females usually receive enough sperm for their entire lifetime from a single insemination. Therefore, release of fertile males does not affect either female population size or disease transmission and can be considered acceptable in all cases. (See "Considerations of Mosquito Genotypes and Phenotypes in MRR" section regarding this issue for both male and female releases.)

Releases that include females. Releases containing females must be considered carefully. Three hazards should be considered as follows: (1) potential to contribute to the population size in the short or long term, (2) direct potential for disease transmission, and (3) introduction of novel genotypes and phenotypes into the wild population.

In contrast to males, female abundance potentially affects the size and possibly the rate of increase of the vector population. A cautious approach is to release only fieldcollected females or larvae from which adults are reared for release at the same location from which they have been collected. However, the practical difficulties, and sometimes impossibility, of collecting a sufficient number of mosquitoes from the field for this purpose will often make it impractical.

Releases of females for MRR, particularly during the early season population-increase phase, should be combined with measures to limit their immediate and delayed impact on population size and potential for increased disease transmission. When the agent that a mosquito might transmit is not present in the environment where the mosquitoes will be released, the effect of a potentially delayed larger population into which an agent might later be introduced could pose risks. In cases of female releases, the collection efforts that will follow release are an important measure to reduce transmission risk.

\section{Considerations of mosquito genotypes and phenotypes in MRR}

While the net numbers of mosquitoes released and collected may be expected to cause no increase or even a reduction in mosquito populations, there remain characteristics of the released mosquitoes that might make their release inadvisable. It is difficult to imagine a treatment of the mosquitoes in the insectary or by marking that would render them harmful (with the possible exception of radioisotope marking which is no longer in use) with the most likely considerations being their phenotypes or genotypes.

Three clear categories should be considered: (1) mosquitoes that are qualitatively and genotypically different from those in the wild-type population where they will be released. An example of a prominent disease vector might be a proposal to release a member of the An. gambiae complex, Anopheles coluzzii, in east Africa where it does not naturally occur. Until 2013, this species was regarded a variant form of $A n$. gambiae s.s. before elevation to formal species status (Coetzee et al. 2013); thus, such a release before this conclusion could easily have been considered as a trivial difference between variants although evidence of differences in their respective larval sites existed (Gimonneau et al. 2012). The fact that An. coluzzii does not occur in east Africa would make such a release essentially the same as releasing an exotic species 
that could become established and potentially affect disease transmission. (2) An insecticide-resistant mosquito presents the possibility of introducing novel resistance alleles into a susceptible wild-type population lacking the resistance mechanism. (3) A mosquito that has a known and possible likelihood to contribute to disease transmission in a way that is significantly different from the study population. Vector attributes of competency and capacity to transmit might include evidence that the mosquito develops unusually high levels of virus or parasites and has extraordinary human feeding attraction or greater longevity.

\section{Guidance for minimizing potential effects of mosquito releases}

While in our assessment, typical experimental releases of mosquitoes as commonly conducted result in minimal hazard, if considered warranted, specific activities can be conducted and conditions implemented that further reduce the risk from experimental releases, even when they contain females.

Commitment and capacity to conduct postrelease collections. Intensive postrelease collection is the most effective means to increase data quality and simultaneously diminish risk. It can result in lower levels of pest biting and potential for disease transmission than if MRR activities had not been conducted. We have described data demonstrating that the numbers of mosquitoes collected as part of the MRR activity are typically greater than the number released. Based on the sheer size of wild populations of mosquitoes compared to the number released and the number of recaptures of both marked and wild mosquitoes, in the majority of cases, normal efforts to recapture mosquitoes will mean that the relatively small numbers of female mosquitoes released in a MRR study will increase neither the local population nor disease transmission. Recapture activities should be described fully in proposals for releases, the trapping methods or devices to be used, and the typical numbers of mosquitoes captured during such activities. Previous data from other surveillance activities may be useful for estimating the numbers that will be recaptured. Those proposing releases should ensure that resources and permissions (including any consent for trap placement and so on) necessary to perform the recapture activities are in place before releases.

Researchers proposing female releases must take measures to ensure that adequate follow-up recaptures are not interfered with. Releases should be avoided if there are factors that might interrupt recaptures, for example, transportation strikes, holidays, staff absences, deteriorating road conditions, or inclement weather.

Control activities might be considered. Some mosquitoes, for example, Ae. aegypti, typically do not move far from point of release. It might be feasible in such cases to consider insecticide spraying activities and larval site destruction or treatment once the postrelease collections have been completed. Such spraying activities would need to be conducted in accordance with local regulations and, if done in and around human habitations, in coordination with proper community engagement and permissions. For mosquitoes that disperse to greater distances on a daily basis, this activity is of less value.

Sexual sterilization might be considered. Sexual sterilization can reduce concerns about novel genotypes and potential for contributing to population increase. However, one of the most common methods of sterilization, irradiation of male mosquitoes, results in reduced vigor and the effect of this on interpretation of the results must be considered. This measure will not eliminate the potential for the released females to transmit pathogens, but it could prevent or reduce the likelihood of the introduction of novel genotypes into the wild population. Due to the deleterious effects on the mosquitoes, this should be considered only as a last resort if other measures are determined to be inadequate, and the effects of genotypic changes are unacceptable. Use of this technique may compromise interpretation of results such that the experiment is not worth conducting.

When biologically relevant results can be obtained, males can be released. With regard to disease transmission and population dynamics, females are of greatest interest and male release information may be useless for the research purpose. In less common cases in which male behavior can provide similar data, only males should be released. Stringent efforts should be made to eliminate contaminating females. We have argued that with the exception of introducing novel genotypes or phenotypes, releases of male mosquitoes will have no detectable effect on either mosquito population growth or disease transmission. Unless there is reason to believe that they differ in some way from the wild mosquitoes that would affect one's ability to control the progeny of such males, these releases can be considered harmless. Testing samples for insecticide resistance from the population to be released and comparing it with samples from the target population could provide a sufficient comparison to evaluate whether novel insecticide resistance mechanisms could be unintentionally introduced into the wild population.

Match the genotypes of the study population. Efforts should be made to match the genotype of the released mosquitoes with those in the study population. Diminished concern is accomplished most easily using mosquitoes collected from the study site for release. Releases consisting of mosquitoes collected at the site where they will be released, particularly of adults and late stage larvae, are unlikely to present a significant hazard compared to no release.

Eliminate biting capacity of released females. Honório et al. (2003) snipped off the proboscises of female Ae. aegypti and Ae. albopictus before their release in Rio de Janeiro to estimate flight ranges for oviposition. This procedure eliminated the possibility that released females could bite hosts, although its effects on dispersal were not tested. Such methods should be carefully considered to ensure that they do not debilitate the mosquitoes that are released to the extent that the results will be invalidated. 


\section{Assessing whether a proposed release activity poses significant risk}

If the answers to the following questions are "yes," the experimental MRR can be considered to have little or no known risk according to the criteria we have described. When the answer is "no," the applicants should be expected to describe the expected risks, which risk mitigation measures will be implemented, and their expected effectiveness.

Does the planned MRR include postrelease collection activities that are likely to collect more females than were released?

Do the mosquitoes to be released originate from the study area and if so, does the proposed release consist only of individuals whose genotype is similar to that of the study population?

Does the proposed release exclude females? If not, how will the investigators ensure that they are disease free?

Is there evidence that the proposed release will not introduce a novel genotype into the population? If not, will the adults be sexually sterile?

\section{Summary}

Typical mosquito MRR activities present little risk when follow-up collections are conducted. Measures are available that further reduce these risks and can be considered in the context of the necessary biological performance characteristics of the mosquitoes. Perhaps more than any other measure, collection activities that occur after releases typically reduce the mosquito populations compared to releases not being performed and provide the greatest assurance of safety.

\section{Acknowledgments}

Publication costs were graciously provided by Target Malaria which receives core funding from the Bill \& Melinda Gates Foundation \& from the Open Philanthropy Project Fund, an advised fund of Silicon Valley Community Foundation.

\section{Author Disclosure Statement}

No competing financial interests exist.

\section{References}

Aboagye-Antwi F, Tripet F. Effects of larval growth condition and water availability on desiccation resistance and its physiological basis in adult Anopheles gambiae sensu stricto. Malar J 2010; 9:225.

Achee NL, Youngblood L, Bangs MJ, Lavery JV, et al. Considerations for the use of human participants in vector biology research: a tool for investigators and regulators. Vector Borne Zoonotic Dis 2015; 15:89-102.

ACME-ASTMH. Arthropod containment guidelines. A project of the American Committee of Medical Entomology and American Society of Tropical Medicine and Hygiene. Vector Borne Zoonotic Dis 2003; 3:61-98.

Amstrup SC, McDonald TL, Manly B, eds. Handbook of Capture-Recapture Analysis. Princeton, NJ: Princeton University Press, 2010.

Bailey NTJ. Improvements in the interpretation of recapture data. J Anim Ecol 1952; 21:120-127.
Birley MH, Charlwood J. The effect of moonlight and other factors on the oviposition cycle of malaria vectors in Madang, Papua New Guinea. Ann Trop Med Parasitol 1989; 83:415-422.

Charlwood J, Alecrim WD. Capture-recapture experiments with Anopheles darlingi in Rondonia, Brazil. Ann Trop Med Parasitol 1989; 83:601-603.

Charlwood J, Dagaro H, Paru R. Blood-feeding and resting behaviour in the Anopheles punctulatus Donitz complex (Diptera, Culicidae) from coastal Papua New Guinea. Bull Entomol Res 1985; 75:463-475.

Charlwood J, Graves PM, Birley MH. Capture-recapture studies with mosquitoes of the group of Anopheles punctulatus Dónitz (Diptera: Culicidae) from Papua New Guniea. Bull Entomol Res 1986; 76:211-227.

Charlwood J, Graves PM, Marshall TF. Evidence for a "memorized" home range in Anopheles farauti females from Papua New Guinea. Med Vet Entomol 1988; 2:101-108.

Charlwood JD. Survival rate variation of Anopheles farauti (Diptera: Culicidae) between neighboring villages in coastal Papua New Guinea. 1986; 23:361-365.

Coetzee M, Hunt RH, Wilkerson R, della Torre A, et al. Anopheles coluzzii and Anopheles amharicus, new members of the Anopheles gambiae complex. Zootaxa 2013; 3619:246-274.

Dame DA, Curtis CF, Benedict MQ, Robinson AS, et al. Historical applications of induced sterilisation in field populations of mosquitoes. Malaria J 2009; 8: Suppl 2:S2.

Edman JD, Scott TW, Costero A, Morrison AC, et al. Aedes aegypti (Diptera: Culicidae) movement influenced by availability of oviposition sites. J Med Entomol 1998; 35:578-583.

Frank JH, Curtis GA. On the bionomics of bromeliad-inhabiting mosquitoes. V. A mark-release-recapture technique for estimation of population size of Wyeomyia vanduzeei. Mosq News 1977; 37:444-452.

Gillies M. Studies on the dispersion and survival of Anopheles gambiae Giles in East Africa, by means of marking and release experiments. Bull Entomol Res 1961; 52:99-127.

Gillies MT, Wilkes TJ. A study of the age-composition of Anopheles gambiae Giles and A. funestus Giles in northeastern Tanzania. Bull Entomol Res 1965; 56:237-262.

Gimonneau G, Pombi M, Choisy M, Morand S, et al. Larval habitat segregation between the molecular forms of the mosquito Anopheles gambiae in a rice field area of Burkina Faso, West Africa. Med Vet Entomol 2012; 26:9-17.

Gu W, Müller G, Schlein Y, Novak RJ, et al. Natural plant sugar sources of Anopheles mosquitoes strongly impact malaria transmission potential. PLoS One 2011; 6:e15996.

Guerra CA, Reiner RC, Perkins TA, Lindsay SW, et al. A global assembly of adult female mosquito mark-release-recapture data to inform the control of mosquito-borne pathogens. Parasit Vectors 2014; 7:276.

Hagler JR, Baker PB, Marchosky R, Machtley SA. Methods to mark termites with protein for mark-release-recapture and mark-capture type studies. Insect Soc 2009; 56:213.

Harrington LC, Françoisevermeylen, Jones JJ, Kitthawee S, Sithiprasasna R, et al. Age-dependent survival of the dengue vector Aedes aegypti (Diptera: Culicidae) demonstrated by simultaneous release-recapture of different age cohorts. J Med Entomol 2008; 45:307-313.

Harrington LC, Scott TW, Lerdthusnee K, Coleman RC, et al. Dispersal of the dengue vector Aedes aegypti within and between rural communities. Am J Trop Med Hyg 2005; 72:209-220.

Honório NA, da Costa Silva W, Leite PJ, Goncalves JM, et al. Dispersal of Aedes aegypti and Aedes albopictus (Diptera: 
Culicidae) in an urban endemic dengue area in the state of Rio de Janeiro, Brazil. Mem Inst Oswaldo Cruz 2003; 98:191-198.

Jones CE, Lounibos LP, Marra PP, Kilpatrick AM. Rainfall influences survival of Culex pipiens (Diptera: Culicidae) in a residential neighborhood in the mid-Atlantic United States. J Med Entomol 2012; 49:467-473.

Lehmann T, Dao A, Yaro AS, Adamou A, et al. Aestivation of the African malaria mosquito, Anopheles gambiae in the Sahel. Am J Trop Med Hyg 2010; 83:601-606.

Lindquist AW, Ikeshoji T, Grab B, de Meillon B, et al. Dispersion studies of Culex pipiens fatigans tagged with ${ }^{32} \mathrm{P}$ in the Kemmendine area of Rangoon, Burma. Bull World Health Organ 1967; 36:21-37.

Lorimer N, Lounibos LP, Petersen JL. Field trials with a translocation homozygote in Aedes aegypti for population replacement. J Econ Entomol 1976; 69:405-409.

Lounibos LP, Lima DC, Lourenco-De-Oliveira R. Prompt mating of released Anopheles darlingi in western Amazonian Brazil. J Am Mosq Control Assoc 1998; 14:210-213.

Maciel-De-Freitas R, Codego CT, Lourenco-De-Oliveira R. Body size-associated survival and dispersal rates of Aedes aegypti in Rio de Janeiro. Med Vet Entomol 2007; 21:284-292.

Mandal S, Sarkar RR, Sinha S. Mathematical models of malaria-a review. Malaria J 2011; 10:202.

McClelland GA, Conway GR. Frequency of blood feeding in the mosquito Aedes aegypti. Nature 1971; 232:485-486.

McDonald PT. Population characteristics of domestic Aedes aegypti (Diptera: Culicidae) in villages on the Kenya Coast II. Dispersal within and between villages. J Med Entomol 1977a; 14:49-53.

McDonald PT. Population characteristics of domestic Aedes aegypti (Diptera: Culicidae) in villages on the Kenya Coast I. Adult survivorship and population size. J Med Entomol 1977b; 14:42-48.

Morris CD, Larson VL, Lounibos LP. Measuring mosquito dispersal for control programs. J Am Mosq Cont Assoc 1991; 7:608-615.

Nayar JK, Provost MW. Quantitative bionomics of Culex nigripalpus (Diptera: Culicidae) populations in Florida: 2. Distribution, dispersal and survival patterns. J Med Entomol 1980; 17:40-50.

Perkins TA, Scott TW, Le Menach A, Smith DL. Heterogeneity, mixing, and the spatial scales of mosquito-borne pathogen transmission. PLoS Comput Biol 2013; 9:e1003327.

Reisen WK, Aslam Y, Siddiqui TF, Khan AQ. A mark-releaserecapture experiment with Culex tritaeniorhynchus Giles. Trans Royal Soc Trop Med Hyg 1978; 72:167-177.

Reisen WK, Bock ME, Milby MM, Reeves WC. Attempted insertion of a recessive autosomal gene into a semi-isolated population of Culex tarsalis (Diptera: Culicidae). J Med Entomol 1985; 22:250-260.
Reisen WK, Lothrop HD. Population ecology and dispersal of Culex tarsalis (Diptera: Culicidae) in the Coachella Valley of California. J Med Entomol 1995; 32:490-502.

Reisen WK, Mahmood F, Azra K. Anopheles culicifacies Giles: adult ecological parameters measured in rural Punjab Province, Pakistan using capture-mark-release-recapture and dissection methods, with comparative observations on An. stephensi Liston and An. subpictus Grassi. Res Pop Ecol 1981; 23:39-60.

Reisen WK, Milby MM, Meyer RP. Population dynamics of adult Culex mosquitoes (Diptera: Culicidae) along the Kern River, Kern County, California, in 1990. J Med Entomol 1992; 29:531-543.

Sheppard PM, MacDonald WW, Tonn RJ, Grab B. The dynamics of an adult population of Aedes aegypti in relation to dengue haemorrhagic fever in Bangkok. J Anim Ecol 1969; 38:661-702.

Silver JB. Mosquito Ecology: Field Sampling Methods, 3rd ed. New York, Springer, 2007:1600.

Taylor CE, Manoukis NC. Effective population size in relation to genetic modification of Anopheles gambiae sensu stricto. In Takken W, Scott TW, eds. Ecological Aspects for Application of Genetically Modified Mosquitoes. Wageningen: Frontis, 2003:133-146.

Trpis M. Adult population estimate of Toxorhynchites brevipalpis. Bull World Health Organ 1973; 48:756-757.

Trpis M, Hausermann W. Dispersal and other population parameters of Aedes aegypti in an African village and their possible significance in epidemiology of vector-borne diseases. Am J Trop Med Hyg 1986; 35:1263-1279.

Trpis M, Hausermann W, Craig GB. Estimates of population size, dispersal, and longevity of domestic Aedes aegypti aegypti (Diptera: Culicidae) by mark-release-recapture in the village of Shauri Moyo in eastern Kenya. J Med Entomol 1995; 32:27-33.

Welch CH, Kline DL, Allan SA, Barnard DR. Laboratory evaluation of a dyed food marking technique for Culex quinquefasciatus (Diptera: Culicidae). J Am Mosq Control Assoc 2006; 22:626-628.

WHO, FNIH. Guidance Framework for Testing of Genetically Modified Mosquitoes. Geneva: WHO, 2014.

Wikelski M, Moskowitz D, Adelman JS, Cochran J, et al. Simple rules guide dragonfly migration. Biol Lett 2014; 2:325-329.

Address correspondence to: Mark Q. Benedict Entomology Branch

Centers for Disease Control and Prevention (CDC) 1600 Clifton Road, MS G49 Atlanta, GA 30333

E-mail: mbenedict@cdc.gov 